\title{
Gravity Induced Anomalies Smearing in Standard Model so that Protons May Never Decay, Except in Black Holes
}

\author{
Stephane H. Maes ${ }^{1}$
}

June 13, 2020

\begin{abstract}
:
Gravity is not modeled in the Standard Model. The Proton decay, predicted by many GUTs, TOEs, supersymmetry, supergravity theories and superstrings, is forbidden in the Standard Model because the proton is the lightest baryon and the conservation of the lepton and baryon numbers would be violated. In New Physics beyond the Standard Model, such violations could be tolerated because the baryon and lepton number symmetries have chirality anomalies. We discuss that in a multi-fold universe, where gravity emerges from entanglement effects, or in a universe where gravity is strong enough to cause chirality flips of fermions, these anomalies are smeared so that the baryon and lepton symmetries could be no more anomalous, and conservation of the baryon and lepton numbers could be seen as stricter. As a result, proton decay could remain forbidden, except when gravity is extreme. It has significant consequences for theories predicting proton decay but matches the total lack of any proton decay observation so far.
\end{abstract}

The new preprint [1] proposes contributions to several open problems in physics like the reconciliation of General Relativity with Quantum Physics, explaining the origin of gravity proposed as emerging from quantum entanglement between particles, detailing contributions to dark matter and dark energy and explaining other Standard Model mysteries without requiring New Physics beyond the Standard Model other than the addition of gravity to the Lagrangian. All this is achieved in a multi-fold universe that may well model our real universe. That is still to be validated.

With the proposed model, spacetime and Physics can be modeled from Planck scales to quantum and macroscopic scales and semi classical approaches appear valid till very small scales. The gravity model recovers General Relativity (GR) at large scale, with massless gravity, but also massive gravity contributions at very small scale, that make gravity no more negligible at these scales.

The hypothetical decay of proton into lighter subatomic particles, such as a neutral Pion and a positron or involving charged Pions or Kaons has been proposed, although forbidden by the Standard Model. Sakharov proposed it as a Baryogenesis, that explains the dominance of matter over anti matter. Theories that try to unify electromagnetic, weak and strong interactions, called Grand Unification Theories, or Unified, (GUTs) typically predict proton decay. Details can be found in [2] (For Proton Decay) and [3] (for GUTs). GUT and Theories of Everything (TOE), have been the long dream of physicists since Einstein encountered then embraced the ideas pioneered by Kaluza and Klein. Most GUTs (there are some exceptions), TOEs, supersymmetry, superstrings, along with super gravity, predict proton decay with different prediction for its half-life.

Yet, unfortunately, experimental results are not good so far: no proton decay has ever been observed and upper bounds for its half-life [2] are raising beyond the predictions of all these theories. Details are discussed in [4]. This has dire consequences for all these theories [5]: they are one by one invalidated or ad hoc changes are needed in order to explain the lack of any observation. Therefore, no GUT or TOE yet. It certainly does not help that most of

\footnotetext{
${ }^{1}$ shmaes.physics@gmail.com
} 
these theories also predict magnetic monopoles: they also have never been observed and [1] also suggests that they might not exist because of gravity.

Going back to the details of the Standard Model, proton decay is forbidden because the proton is the lightest baryon and the Standard Model conserves the baryon and lepton numbers, as result of symmetries. Yet it is known that these symmetries are anomalous, meaning that they create problems when the classical or non-quantized formulations are quantized. As a result, they is not associated to interactions, fields or boson carriers. The difference between these numbers however cancels the anomalies and it could be associated to a boson (in models beyond the Standard Model). The anomalies involve the asymmetries in chirality: left-handed and righthanded particles do not interact the same way in the Standard Model $[6,7]$.

With its proposal for gravity emergence from entanglement, [1] shows that gravity can be added to the Standard Model without really introducing the New Physics brought by all these other theories that predict proton decay. In particular, [1] shows that fermions (massive and massless) have their chirality flipped (back and forth between left-handed and right-handed) by gravity. This allows [1] to propose a new model to explain the mass of the neutrino that also does not require New Physics. In the present case, these flips can be seen as allowing particles to be at time right-handed and at time left-handed, effects appearing at very small scales, where massive gravity plays a role. As a result, we observe a smeared average, that will not care of the difference of behavior between left-handed and right-handed particles, as all are sometimes in one mode and sometimes in the other. Doing so, could eliminate the anomalies by canceling the problematic axial currents, e.g. just as in a similar effect in QCD involving QCD sphalerons [11]. Of course, it is a conjecture, that requires more formal work to validate if such a smearing concept would make sense for anomalies and what is exactly its impact. An immediate consequence would be that, because the baryon and lepton numbers are now more stringently conserved, there is no easy leeway to break these symmetries and have proton decays. This is a very important result not limited to the model of [1]: gravity may eliminate chirality anomalies in symmetries of the Standard Model in all models where gravity induces chirality flips for fermions.

Therefore, if [1] is right, proton decay could be forbidden by more fundamental and unbroken (i.e. not anomalous) symmetries and may never be observed (in normal conditions of reasonable gravity). Of course, the reasoning still need to be peer reviewed and it also relies on the proposal that the smearing or averaging saves the symmetries in Physics.

It creates hurdles for any theory that predicts proton decay, and we saw that this includes most of the GUTs, TOEs, and superstring models. For non-TOEs, their best way out would probably be to argue that their prediction is due to the absence of gravity in their model and that they would probably also drop their predictions or estimates for proton decays if gravity is correctly added. Yet, that may not work for models that are already encompassing gravity or gravitons (e.g. superstrings). This is not the argument encountered usually today [8].

[1] also mentions that, in a massive macroscopic black hole, the quarks can probably be compressed till they fully overlap and annihilate their colors. And so, yes, proton decay can take place, but only in black holes, or extreme gravity, and with half-life way larger than anything predicted by conventional theories.

If proton decay was to be observed, way beyond the predictions of GUTs and TOEs, then [1] could provide an explanation with its strong (massive) gravity contribution proposal. Depending on the resulting observed half-life of the proton, it may explain the large half time (due to the extreme gravity effects), or if invalidated (i.e. the halftime is within expectations of a conventional GUT or TOE), it would be only create problems for the anomaly smearing hypothesis of [1]: it did not prevent the anomalies of the baryon and lepton number symmetries. Note that whatever is the outcome, proton decay should not be seen as a criteria to invalidate the model of gravity emergence from entanglement and multi-fold mechanisms proposed in [1].

In conclusion, [1] provides a new hypothesis to explain why, with gravity, proton decay has never been observed under normal conditions over the last $40+$ years. It also throw new challenges to the theories that predict proton decay, especially if they also already claim to model gravity. 
It is also worth noting that, even without the models of [1], if gravity can be shown to play a strong enough role at very small scale so that it can flip chirality of fermions, the arguments above can be repeated in such a context and they would result into the same consequences for proton decay. [1] has just the convenient advantage of already making a case for why gravitation would be strong enough to cause the chirality flips.

This paper really contributed two things: i) the considerations in the context of [1]. ii) A new theoretical hypothesis on how and why a strong enough gravity at small scales can remove the anomaly from baryon and lepton number symmetries. That is new in itself and should be considered, no matter what the outcome of investigation of concept of multi-fold universes proposed in [1] are. In both cases, it is proposed that removing the anomalies, could render proton decay less acceptable because of gravity. It also exemplifies and makes a case for adding gravity consideration in the Standard Model. [1] discusses other examples where gravity could matter to the Standard Model.

The analysis presented in this paper and in [1] are quite different analyses of the gravity effects than what is conventionally proposed (e.g. see [8] for a review), where the effects are also seen as smearing, but rather in terms of impact on the coupling constants and fine structures constants; with recognition that gravity may significantly affect the proton decay and half-life estimates but without identifying that it may instead eliminate its possibility altogether.

Interactions with Black holes and magnetic monopoles have also been proposed as sources of proton decay [9,10]. [1] settles the magnetic monopole by arguing that they do not exist, also because of gravity effects. Black holes are discussed above. [1] models particles and spacetime as microscopic black holes. It leads to a distinction between business as usual for microscopic black holes (i.e. just think of particle interactions) and the effects within macroscopic black holes able to compress quarks and annihilate colors. In a multi-fold universe proposed in [1], extremal black holes can disintegrate into smaller black holes and eventually into protons, themselves as compositions of extremal black holes (quarks) and other elementary particle black holes. This way, there should be no pesky remnant troubles.

In retrospect, the chirality flipping, and semi classical suitability found in [1], were really key in coming up with the new analysis proposed here. But the reasoning indicates that it should be possible to apply the outcome to our real universe not only if is well modeled by [1] but also if it is not the case with suitable gravity effects that flip chirality.

References: (most references come from popular science to make the discussion more approachable)

[1]: Stephane H. Maes, (2020) “Quantum Gravity Emergence from Entanglement in a Multi-Fold

Universe", viXra:2006.0088v1, (June 9, 2020).

[2]: https://en.wikipedia.org/wiki/Proton decay

[3]: https://en.wikipedia.org/wiki/Grand_Unified Theory

[4]: https://www.forbes.com/sites/startswithabang/2020/01/03/how-certain-are-we-that-protons-dontdecay/\#762c60c43544

[5]: https://www.quantamagazine.org/no-proton-decay-means-grand-unification-must-wait-20161215/

[6]: Schwartz, M.D., (2013), "Quantum Field Theory and the Standard Model", Cambridge University Press.

[7]: Kazuo Fujikawa, Hiroshi Suzuki, (2014), "Path Integrals and Quantum Anomalies", Oxford University Press, USA; Reprint edition (January 21, 2014).

[8]: Pran Nath, Pavel Fileviez Perez, (2006), "Proton stability in grand unified theories, in strings, and in branes", arXiv:hep-ph/0601023v3.

[9]: Steven Weinberg, (1981), "The Decay of the Proton", Scientific American, Vol. 244, No. 6 (June 1981), pp. 64-

75.

[10]: https://www.npl.washington.edu/AV/altvw01.html 
[11]: Steven D. Bass, (2004), "Anomalous commutators and electroweak baryogenesis", arXiv:hep-ph/0403219v1 